\title{
Charged-impurity scattering in graphene
}

\author{
J.-H. CHEN ${ }^{1,2,3 *}$, C. JANG ${ }^{1,2,3 *}$, S. ADAM ${ }^{2,3,4}$, M. S. FUHRER ${ }^{1,2,3}$, E. D. WILLIAMS ${ }^{1,2,3,5,6}$ \\ AND M. ISHIGAM|2,3†
}

\begin{abstract}
${ }^{1}$ Materials Research Science and Engineering Center; ${ }^{2}$ Physics Department; ${ }^{3}$ Center for Nanophysics and Advanced Materials; ${ }^{4}$ Condensed Matter Theory Center; ${ }^{5}$ Laboratory for Physical Sciences; ${ }^{6}$ Institute for Physical Science and Technology, University of Maryland, College Park, Maryland 20742, USA

*These authors contributed equally to this work

${ }^{\dagger}$ Present address: Department of Physics, University of Central Florida, 4000 Central Florida Boulevard, Orlando, Florida 32816-2385, USA

†e-mail: ishigami@physics.ucf.edu
\end{abstract}

Since the initial demonstration of the ability to experimentally isolate a single graphene sheet ${ }^{1}$, a great deal of theoretical work has focused on explaining graphene's unusual carrierdensity-dependent conductivity $\sigma(n)$, and its minimum value $\left(\sigma_{\text {min }}\right)$ of nearly twice the quantum unit of conductance $\left(4 e^{2} / h\right)$ (refs 1-6). Potential explanations for such behaviour include short-range disorder ${ }^{7-10}$, 'ripples' in graphene's atomic structure $^{11,12}$ and the presence of charged impurities ${ }^{7,8,13-18}$. Here, we conduct a systematic study of the last of these mechanisms, by monitoring changes in electronic characteristics of initially clean graphene ${ }^{19}$ as the density of charged impurities $\left(n_{\text {imp }}\right)$ is increased by depositing potassium atoms onto its surface in ultrahigh vacuum. At non-zero carrier density, charged-impurity scattering produces the widely observed linear dependence ${ }^{1-6}$ of $\sigma(n)$. More significantly, we find that $\sigma_{\min }$ occurs not at the carrier density that neutralizes $n_{\text {imp }}$, but rather the carrier density at which the average impurity potential is zero ${ }^{15}$. As $n_{\text {imp }}$ increases, $\sigma_{\min }$ initially falls to a minimum value near $4 e^{2} / h$. This indicates that $\sigma_{\min }$ in the present experimental samples ${ }^{1-6}$ is governed not by the physics of the Dirac point singularity ${ }^{20,21}$, but rather by carrier-density inhomogeneities induced by the potential of charged impurities ${ }^{6,8,14,15}$.

Several theoretical studies ${ }^{7,8,13-15,17,18}$ have predicted chargedimpurity scattering in graphene to produce $\sigma(n)$ of the form

$$
\sigma(n)=C e\left|\frac{n}{n_{\text {imp }}}\right|+\sigma_{\text {res }},
$$

where $C$ is a constant, $e$ is the electronic charge and $\sigma_{\text {res }}$ is the residual conductivity at $n=0$ (this last term was predicted only in refs 17,18; see the Supplementary Information for a more detailed discussion of the theory). Hwang et al. ${ }^{14}$ first calculated the screened Coulomb potential within the random phase approximation, and used the results to determine $C=5 \times 10^{15} \mathrm{~V}^{-1} \mathrm{~s}^{-1}$. Novikov ${ }^{16}$ noted that, beyond the Born approximation used in ref. 14, an asymmetry in $C$ for attractive versus repulsive scattering (electron versus hole carriers) is expected for Dirac fermions. Experimentally, the behaviour described by equation (1) is ubiquitously observed ${ }^{1-6}$ in graphene, strongly suggesting that charged-impurity scattering is the dominant scattering mechanism in present samples. Here, we provide the first direct verification of equation (1) for charged-impurity scattering in graphene, and determine the constant $C$. We also observe the expected asymmetry for attractive versus repulsive scattering for Dirac fermions ${ }^{16}$.
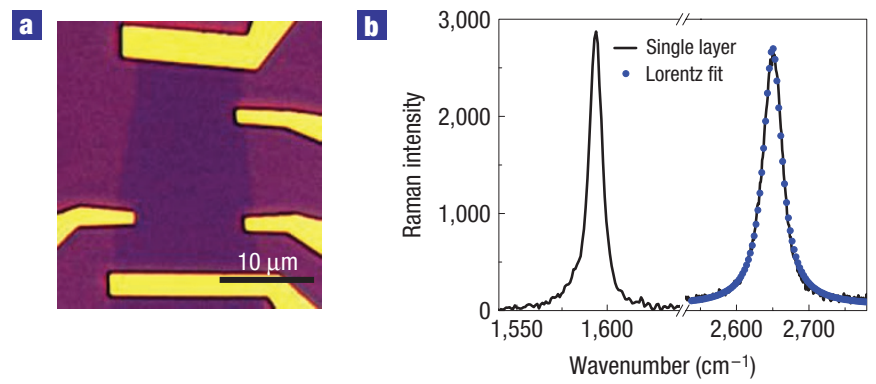

Figure 1 Graphene device. a, Optical micrograph of the device. $\mathbf{b}, 633 \mathrm{~nm}$ micro-Raman shift spectrum acquired over the device area, with lorentzian fit to the $\mathrm{D}^{\prime}$ peak, confirming that the device is made from single-layer graphene (vertical scale is the same throughout $\mathbf{b}$ ).

At low carrier density, the conductivity does not vanish linearly, but rather saturates to a constant value near $4 e^{2} / h$ (ref. 2). Early theoretical work ${ }^{20,21}$ on massless Dirac fermions predicted $\sigma_{\min }=4 e^{2} / \pi h$ for vanishing disorder. However, in the presence of charged impurities, a finite conductivity $\sim 4 e^{2} / h$ is predicted over a plateau of width $\Delta V_{\mathrm{g}}$ (refs $\left.8,14,15\right)$. Here, we measure experimentally the dependence on $n_{\text {imp }}$ of $\sigma_{\min }, \Delta V_{\mathrm{g}}$ and the gate voltage $V_{\mathrm{g}, \min }$ at which the minimum conductivity occurs, and find agreement with theoretical predictions ${ }^{8,14,15}$, indicating that disorder due to charged impurities is the relevant physics at the minimum conductivity point in present samples.

Figure la shows the graphene device used in this study, and Fig. $1 \mathrm{~b}$ shows its micro-Raman spectrum; the single lorentzian $\mathrm{D}^{\prime}$ peak confirms that the device is single-layer graphene ${ }^{22}$ (see the Methods section). To vary the density of charged impurities, the device was dosed with a controlled potassium flux in sequential $2 \mathrm{~s}$ intervals at a sample temperature $T=20 \mathrm{~K}$ in ultrahigh vacuum (UHV). The gate-voltage-dependent conductivity $\sigma\left(V_{\mathrm{g}}\right)$ was measured in situ for the pristine device, and again after each doping interval. After several doping intervals, the device was annealed in UHV to $490 \mathrm{~K}$ to remove weakly adsorbed potassium ${ }^{23}$, then cooled to $20 \mathrm{~K}$ and the doping experiment repeated; four such runs (runs 1-4) were carried out in total.

Figure 2 shows the conductivity versus gate voltage for the pristine $^{19}$ device and at three different doping concentrations at $20 \mathrm{~K}$ in UHV for run 3 (see also the Supplementary Information 


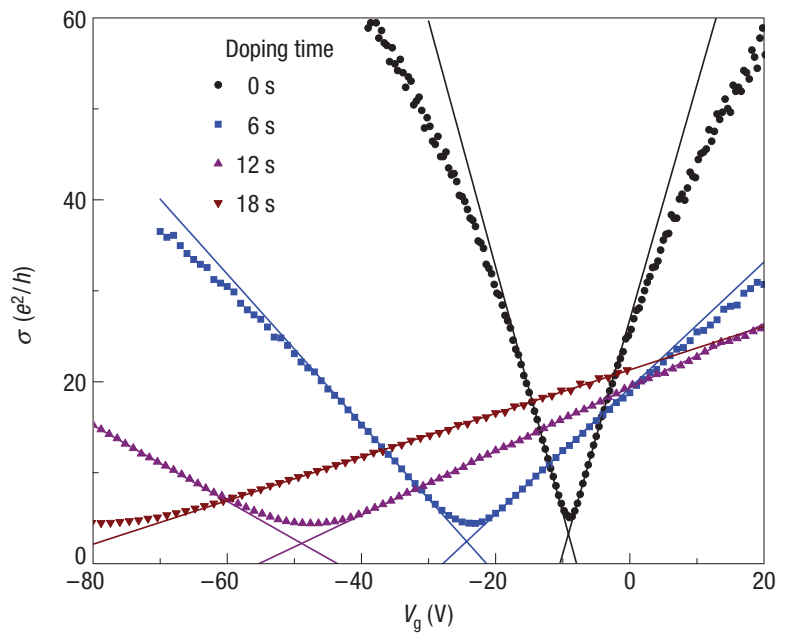

Figure 2 Potassium doping of graphene. The conductivity $(\sigma)$ versus gate voltage $\left(V_{g}\right)$ curves for the pristine sample and three different doping concentrations taken at $20 \mathrm{~K}$ in UHV. Data are from run 3. Lines are fits to equation (1), and the crossing of the lines defines the points of the residual conductivity and the gate voltage at minimum conductivity $\left(\sigma_{\text {res }}, V_{g, \text { min }}\right)$ for each data set. The variation of $\sigma_{\min }$ with impurity concentration is shown in Fig. 5 .

for measurements on a second device). On K-doping, (1) the mobility decreases, (2) $\sigma\left(V_{\mathrm{g}}\right)$ becomes more linear, (3) the mobility asymmetry for holes versus electrons increases, (4) the gate voltage of minimum conductivity $V_{g \text {,min }}$ shifts to more negative gate voltage, (5) the width of the minimum conductivity region in $V_{\mathrm{g}}$ broadens and (6) the minimum conductivity $\sigma_{\min }$ decreases, at least initially (see also Fig. 5 below and Supplementary Information). In addition, (7) the linear $\sigma\left(V_{\mathrm{g}}\right)$ curves extrapolate to a finite $\sigma_{\text {res }}$ at $V_{\mathrm{g}, \min }$. All of these features have been predicted ${ }^{7,8,13-15,17,18}$ for charged-impurity scattering in graphene; we will discuss each in detail below.

Effects (4) and (5) were observed in a previous study in which graphene was exposed to molecular species ${ }^{24}$. However, the authors reported no changes in mobility, concluding that chargedimpurity scattering contributes negligibly to the mobility of graphene. As discussed further in the Supplementary Information, the previous experiments did not control the environment and had low initial sample mobility. The failure to observe effects (1)-(3) therefore is most likely due to the presence of significant concentrations of both positively and negatively charged impurities $^{24,25}$, although the presence of water and resist residue ${ }^{19}$ may also be contributing factors ${ }^{24}$.

We first examine the behaviour of $\sigma\left(V_{\mathrm{g}}\right)$ at high carrier density. For $V_{\mathrm{g}}$ not too near $V_{\mathrm{g}, \min }$, the conductivity can be fitted (Fig. 2) by

$$
\sigma\left(V_{\mathrm{g}}\right)= \begin{cases}\mu_{\mathrm{e}} c_{\mathrm{g}}\left(V_{\mathrm{g}}-V_{\mathrm{g}, \text { min }}\right)+\sigma_{\text {res }} & V_{\mathrm{g}}>V_{\mathrm{g}, \text { min }} \\ -\mu_{\mathrm{h}} c_{\mathrm{g}}\left(V_{\mathrm{g}}-V_{\mathrm{g}, \text { min }}\right)+\sigma_{\text {res }} & V_{\mathrm{g}}<V_{\mathrm{g}, \text { min }}\end{cases}
$$

where $\mu_{\mathrm{e}}$ and $\mu_{\mathrm{h}}$ are the electron and hole field-effect mobilities, $c_{\mathrm{g}}$ is the gate capacitance per unit area, $1.15 \times 10^{-4} \mathrm{~F} \mathrm{~m}^{-2}$, and $\sigma_{\text {res }}$ is the residual conductivity that is determined by the fit. The mobilities are reduced by an order of magnitude during each run, and recover on annealing. The electron mobilities ranged from 0.081 to $1.32 \mathrm{~m}^{2} \mathrm{~V}^{-1} \mathrm{~s}^{-1}$ over the four runs, nearly covering the range of mobilities reported so far in the literature $\left(\sim 0.1-2 \mathrm{~m}^{2} \mathrm{~V}^{-1} \mathrm{~s}^{-1}\right)($ refs $2,3,6)$.

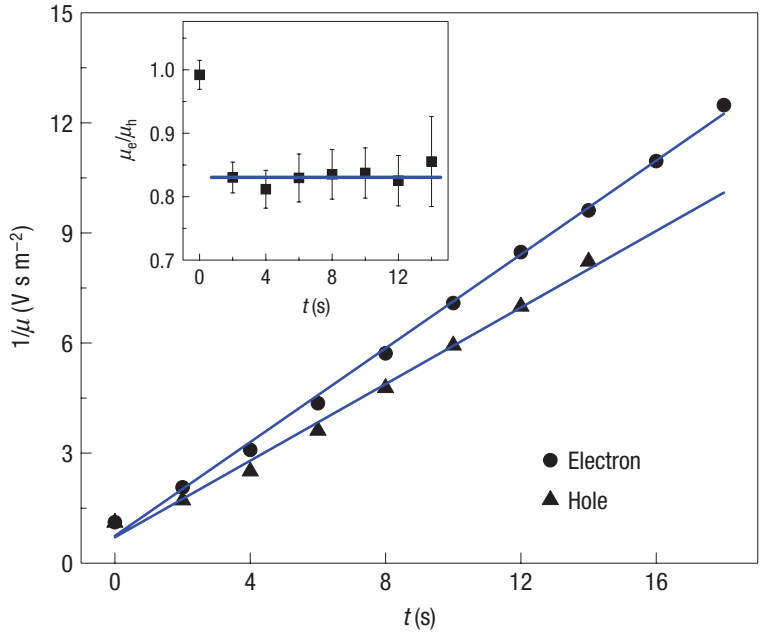

Figure 3 Inverse of electron mobility $1 / \mu_{\mathrm{e}}$ and hole mobility $1 / \mu_{\mathrm{h}}$ versus doping time. Experimental error determined from standard error propagation is less than $4 \%$ (see the Methods section). Lines are linear fits to all data points. Inset: The ratio of $\mu_{\mathrm{e}}$ to $\mu_{\mathrm{h}}$ versus doping time. Error bars represent experimental error in determining the mobility ratio from the fitting procedure (see the Methods section). Data are from run 3 (same as Fig. 2).

For uncorrelated scatterers, the mobility depends inversely on the density of charged impurities, $1 / \mu \propto n_{\text {imp }}$, and equations (1) and (2) are identical. We assume $n_{\mathrm{imp}}$ varies linearly with dosing time $t$ as potassium is added to the device. Figure 3 shows $1 / \mu_{\mathrm{e}}$ and $1 / \mu_{\mathrm{h}}$ versus $t$, which are linear, in agreement with $1 / \mu \propto n_{\text {imp }}$, hence verifying that equation (1) describes charged-impurity scattering in graphene. We estimate the dosing rate $\mathrm{d} n_{\text {imp }} / \mathrm{d} t=(2.6-3.2) \times 10^{15} \mathrm{~m}^{-2} \mathrm{~s}^{-1}$ and the maximum concentration of $(1.4-1.8) \times 10^{-3}$ potassium per carbon (see the Supplementary Information). From this point, we parameterize the data by $1 / \mu_{\mathrm{e}}$, proportional to the impurity concentration (the data set for $\mu_{\mathrm{e}}$ is more extensive than for $\mu_{\mathrm{h}}$ because of the limited $V_{\mathrm{g}}$ range accessible experimentally).

Figure 3 , inset shows that, although the $\mu_{\mathrm{e}}$ and $\mu_{\mathrm{h}}$ are not identical, their ratio is fairly constant at $\mu_{\mathrm{e}} / \mu_{\mathrm{h}}=0.83 \pm 0.01$ (see the Methods section). Novikov ${ }^{16}$ predicted $\mu_{\mathrm{e}} / \mu_{\mathrm{h}}=0.37$ for an impurity charge $Z=1$; however, the asymmetry is expected to be reduced when screening by conduction electrons is included.

As K-dosing increases and mobility decreases, the linear behaviour of $\sigma\left(V_{\mathrm{g}}\right)$ (Fig. 2) associated with charged-impurity scattering dominates, as predicted theoretically ${ }^{14}$. At the lowest $\mathrm{K}$-dosing level, sublinear behaviour is observed for large $\left|V_{\mathrm{g}}-V_{\mathrm{g}, \text { min }}\right|$ as anticipated. The dependence of the conductivity on carrier density $n \propto\left|V_{\mathrm{g}}-V_{\mathrm{g}, \text { min }}\right|$ is expected to be $\sigma \propto n^{a}$ with $a=1$ for charged impurities and $a<1$ for short-range and ripple scattering (see the Supplementary Information). Adding conductivities in inverse according to Matthiessen's rule indicates that scattering other than by charged impurities will dominate at large $n$, with the crossover occurring at larger $n$ as $n_{\text {imp }}$ is increased $^{14}$. A previous study ${ }^{3}$ also found more linear $\sigma\left(V_{g}\right)$ for devices with lower mobility. Thus, our data indicate that the variation in observed field-effect mobilities of graphene devices is determined by the level of unintentional charged impurities.

We now examine the shift of the curves in $V_{g}$. Figure 4 shows $V_{\mathrm{g}, \min }$ as a function of $1 / \mu_{\mathrm{e}}$. Run 1 differs from runs 2-4, presumably owing to irreversible changes as potassium reacts with charge traps on silicon oxide and/or edges and defects of the 


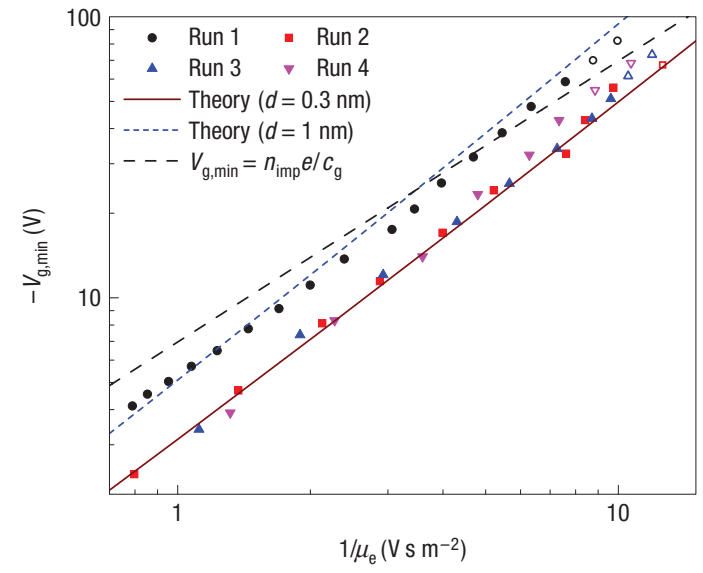

Figure 4 Shift of minimum conductivity point with doping. The gate voltage of minimum conductivity $V_{g, \text { min }}$ as a function of inverse mobility, which is proportional to the impurity concentration. All four experimental runs are shown. Each data set has been shifted by a constant offset in $V_{g, \text { min }}$ to make $V_{g, \text { min }}\left(1 / \mu_{\mathrm{e}} \rightarrow 0\right)=0$, to account for any rigid threshold shift. The offset (in volts) is $-10,3.1,5.6$ and 8.2 for the four runs, respectively, with the variation likely to be due to accumulation of $K$ in the $\mathrm{Si}_{2}$ on successive experiments. The open circles are $V_{\mathrm{g} \text {.min }}$ obtained directly from the $\sigma\left(V_{g}\right)$ curves rather than fits to equation (1) because the linear regime of the hole side of these curves is not accessible owing to heavy doping. The solid and short-dashed lines are from the theory of Adam et $a l .^{15}$ for an impurity-graphene distance $d=0.3 \mathrm{~nm}$ (solid line) and $d=1 \mathrm{~nm}$ (short-dashed line), and approximately follow power laws with slopes 1.2 and 1.3, respectively. The long-dashed line shows the linear relationship $\Delta V_{\mathrm{g}, \min }=n_{\mathrm{imp}} Z e / c_{\mathrm{g}}$, where $n_{\text {imp }}=\left(5 \times 10^{15} \mathrm{~V}^{-1} \mathrm{~s}^{-1}\right) / \mu$ and $Z=1$.

graphene sheet. After run 1, subsequent runs are very repeatable, other than an increasing rigid shift to more negative voltage in the initial gate voltage of minimum conductivity. (The same distinction between first and subsequent experiments is seen in Fig. 5 as well.) It might be expected that the minimum conductivity would occur at the induced carrier density that precisely neutralizes the charged-impurity density: $n=-Z n_{\text {imp }}$ or $\Delta V_{\mathrm{g}, \min }=-n_{\mathrm{imp}} Z e / c_{\mathrm{g}}$ (ref. 24), where $e$ is the elementary charge and $Z e$ is the charge of the potassium ion. This prediction is shown as the long-dashed line in Fig. 4; the experimental data show a distinctly different effective power-law dependence. Adam et al. ${ }^{15}$ proposed that the minimum conductivity in fact occurs at the added carrier density $\bar{n}$ at which the average impurity potential is zero, $\Delta V_{\mathrm{g}, \min }=-\bar{n} e / c_{\mathrm{g}}$, where $\bar{n}$ is a function of $n_{\text {imp }}$, the impurity spacing $d$ from the graphene plane and the dielectric constant of the $\mathrm{SiO}_{2}$ substrate. The theory also assumes that $Z=1$; experimentally, a reasonable evaluation ${ }^{26}$ of $Z$ for dilute potassium on graphite is $\sim 0.7$ (see the Supplementary Information for further discussion of $Z \neq 1$ ). The theoretical lines in Fig. 4 are given by the exact result of Adam et $a .^{15}$, and follow an approximate power-law behaviour of $\Delta V_{\mathrm{g}, \min } \propto n_{\mathrm{imp}}^{b}$ with $b=1.2-1.3$, which agrees well with experiment. The only adjustable parameter is the impurity-graphene distance $d$; we show the results for $d=0.3 \mathrm{~nm}$ (a reasonable value for the distance of potassium on graphene $\mathrm{e}^{26-28}$ ) and $d=1.0 \mathrm{~nm}$ (the value used by Adam et al.). As $\Delta V_{\mathrm{g}, \min }$ gives an independent estimate of $n_{\mathrm{imp}}$, the quantitative agreement in Fig. 4 verifies that $C=5 \times 10^{15} \mathrm{~V}^{-1} \mathrm{~s}^{-1}$ in equation (1), as expected theoretically.

We now turn to the behaviour near the point of minimum conductivity. Figure 5 a shows the minimum conductivity $\sigma_{\min }$ and residual conductivity $\sigma_{\text {res }}$ as a function of $1 / \mu_{\mathrm{e}}$, and Fig. 5b shows the plateau width $\Delta V_{\mathrm{g}}$ as a function of $1 / \mu_{\mathrm{e}} ; \Delta V_{\mathrm{g}}$ is the
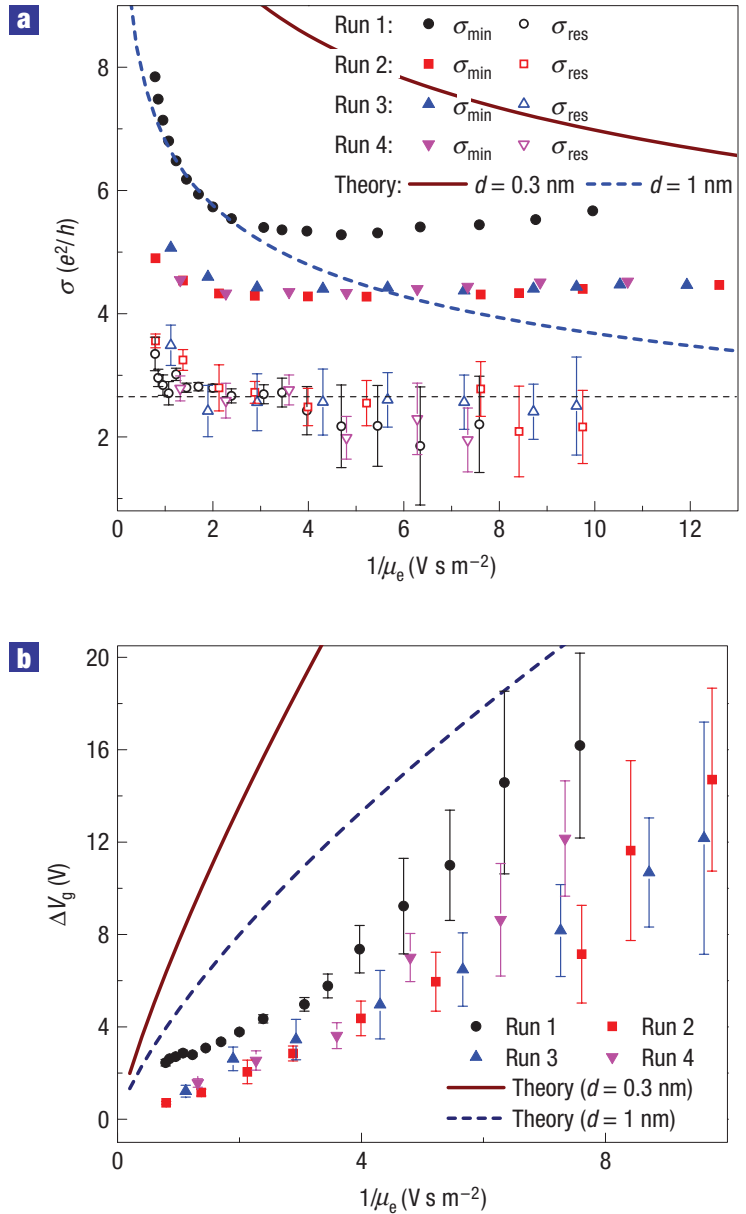

Figure 5 Change in behaviour near the minimum conductivity point with doping. a, The minimum conductivity and the residual conductivity (defined in text) as a function of $1 / \mu_{\mathrm{e}}$ (proportional to the impurity density). $\mathbf{b}$, The plateau width $\Delta V_{\mathrm{g}}$ as a function of $1 / \mu_{\mathrm{e}}$. In $\mathbf{a}$ and $\mathbf{b}$, data from all four experimental runs are shown, as well as the theoretical predictions of the minimum conductivity and plateau width from Adam et al. ${ }^{15}$ for $d=0.3 \mathrm{~nm}$ (solid line) and $d=1 \mathrm{~nm}$ (short-dashed line). Error bars represent experimental error in determining $\sigma_{\text {res }}$ and $\Delta V_{g}$ from the fitting procedure (see the Methods section); $\sigma_{\text {min }}$ is measured directly.

difference between the two values of $V_{\mathrm{g}}$ for which $\sigma_{\min }=\sigma\left(V_{\mathrm{g}}\right)$ in equation (2). The predictions from the theory of Adam et al. ${ }^{15}$ for $\sigma_{\text {min }}$ and $\Delta V_{\mathrm{g}}$ are also shown. The minimum conductivity drops on initial potassium dosing, and shows a broad minimum near $4 e^{2} / h$ before gradually increasing with further exposure. Notably, the cleanest samples show $\sigma_{\min }$ significantly greater than $4 e^{2} / h$, and strongly dependent on charged-impurity density, indicating that the universal behaviour ${ }^{20,21}$ of $\sigma_{\min }$ associated with the Dirac point is not observed even in the cleanest samples. The irreversible change in the value of $\sigma_{\text {min }}$ between run 1 and runs $2-4$ is larger than the entire variation within runs $2-4$. This difference between initial and subsequent runs indicates that the initial K-dosing and anneal cycle introduces other types of disorder (possibly short-range scatterers induced by irreversible chemisorption of potassium on defects or reaction of potassium with adsorbates) that have a comparable or greater impact on $\sigma_{\min }$ than charged impurities. That, for some disorder conditions (run 1), $\sigma_{\min }$ varies significantly with $n_{\text {imp }}$, but for other conditions (runs 2-4), the decrease in $\sigma_{\min }$ saturates rapidly with increasing $n_{\text {imp }}$, and is nearly constant for a very broad 
range of doping, suggests that the substantial variations reported in the literature (some groups report that $\sigma_{\min }$ is a universal value $^{2}$, whereas other groups observe variation in $\sigma_{\min }$ from sample to sample ${ }^{3}$ ) are probably due to poor control of the chemical environment of the devices measured. The observed residual conductivity $\sigma_{\text {res }}$ is finite and surprisingly constant (Fig. 5a); it is only weakly dependent on doping, and shows little variation between the first run and subsequent runs. Finite $\sigma_{\text {res }}$ has been predicted theoretically ${ }^{17,18}$ for graphene with charged impurities; however, the magnitude has not been calculated. The change of $\Delta V_{\mathrm{g}}$ with doping (Fig. 5b) agrees only qualitatively with the theory, which predicts larger values and a sublinear dependence on doping. However, the quantitative disagreements between experiment and theory in Fig. $5 \mathrm{a}, \mathrm{b}$ are connected: mobility, minimum conductivity and residual conductivity determine $\Delta V_{\mathrm{g}}$.

In summary, the dependence of conductivity of graphene on the density of charged impurities has been demonstrated by controlled potassium doping of clean-graphene devices in UHV at low temperature. The minimum conductivity depends systematically on charged-impurity density, decreasing on initial doping, and reaching a minimum near $4 e^{2} / h$ only for non-zero chargedimpurity density, indicating that the universal conductivity at the Dirac point ${ }^{2,20,21}$ has not yet been probed experimentally. The high-carrier-density conductivity is quantitatively consistent with theoretical predictions for charged-impurity scattering in graphene ${ }^{7,8,13-15,17,18}$. The addition of charged impurities produces a more linear $\sigma\left(V_{\mathrm{g}}\right)$, and reduces the mobility, with the constant $C=\mu n_{\text {imp }}=5 \times 10^{15} \mathrm{~V}^{-1} \mathrm{~s}^{-1}$, in excellent agreement with theory. The asymmetry for repulsive versus attractive scattering predicted for massless Dirac quasiparticles ${ }^{16}$ is observed for the first time. Finally, the minimum conductivity point ${ }^{15}$ occurs at the applied gate voltage at which the average impurity potential is zero and not at the voltage at which the gate-induced carrier density neutralizes the impurity charge.

Other observations indicate the need for fuller experimental and theoretical understanding. The irreversible changes in the behaviour around $V_{\mathrm{g} \text {,min }}$ between the first and subsequent doping runs indicate that the precise value of the minimum conductivity depends on the interplay of more than one type of disorder, and hence cannot be explained by existing theories ${ }^{7-10,12,14,15,17,18}$. An interesting new feature, the residual conductivity, may point to physics beyond the simple Boltzmann transport picture ${ }^{17,18}$. Further experiments including introducing short-range (neutral) scatterers to graphene will be useful in addressing these questions. Full understanding may require scanned-probe studies of graphene under well-controlled environmental conditions ${ }^{19}$, which can completely characterize the disorder due to defects, charged and neutral adsorbates and ripples, as well as probe the electron scattering from each ${ }^{29}$.

\section{METHODS}

Graphene is obtained from Kish graphite by mechanical exfoliation ${ }^{30}$ on $300 \mathrm{~nm}$ $\mathrm{SiO}_{2}$ over doped $\mathrm{Si}$ (back gate), with $\mathrm{Au} / \mathrm{Cr}$ electrodes defined by electron-beam lithography (Fig. 1a). Raman spectroscopy confirms that the samples are singlelayer graphene $e^{22}$ (Fig. 1b). After fabrication, the devices are annealed in $\mathrm{H}_{2} / \mathrm{Ar}$ at $300^{\circ} \mathrm{C}$ for $1 \mathrm{~h}$ to remove resist residues ${ }^{19}$. Gas flows are $1,700 \mathrm{ml} \mathrm{min}^{-1}\left(\mathrm{H}_{2}\right)$ and $1,900 \mathrm{ml} \mathrm{min}^{-1}(\mathrm{Ar})$ at $1 \mathrm{~atm}$; gases are flowing throughout heating and cooling. The devices are mounted on a liquid-helium-cooled cold finger in a UHV chamber, so that the temperature of the device can be controlled from 20 to $490 \mathrm{~K}$.

Following a vacuum bakeout, each device is annealed in UHV at $490 \mathrm{~K}$ overnight to remove residual adsorbed gases. Experiments are carried out at pressures lower than $5 \times 10^{-10}$ torr and device temperature $T=20 \mathrm{~K}$. Potassium doping is accomplished by passing a current of $6.5 \mathrm{~A}$ through a getter (SAES Getters) for $40 \mathrm{~s}$ before the shutter is opened for $2 \mathrm{~s}$. The getter temperature during each potassium dosage was $763 \pm 5 \mathrm{~K}$ as measured by optical pyrometry. The stability of the potassium flux was monitored by a residual gas analyser positioned off-axis and behind the sample (see the Supplementary Information). All measurements were carried out on one four-probe device shown in Fig. 1a, although several two-probe devices showed similar behaviour (see Supplementary Information, Figs S1,S3-S5).

Conductivity $\sigma$ is determined from the measured four-probe sample resistance $R$ using $\sigma=(L / W)(1 / R)$. Because the sample is not an ideal Hall bar, there is some uncertainty in the (constant) geometrical factor $L / W$. We estimate $L / W=0.80 \pm 0.09$, where the error bars represent \pm one standard deviation. This $11 \%$ uncertainty in $L / W$ translates into an $11 \%$ uncertainty in the vertical axes of Figs 2 and 3, the horizontal axes of Figs 4 and $5 \mathrm{~b}$ and both axes of Fig. 5a. Such scale changes are comparable to the spread among different experimental runs, and do not alter our conclusions. Notably, the uncertainty represents a systematic error, so relative changes in, for example, the minimum conductivity with charged-impurity density are still correct.

Best fits to equation (1) were determined using a least-squares linear fit to the steepest regime in the $\sigma\left(V_{\mathrm{g}}\right)$ curves. The steepest regime of the $\sigma\left(V_{\mathrm{g}}\right)$ curves was determined by examining $\mathrm{d} \sigma / \mathrm{d} V_{\mathrm{g}}$; the fit was carried out over a $2 \mathrm{~V}$ interval in $V_{\mathrm{g}}$ around the maximum of $\mathrm{d} \sigma / \mathrm{d} V_{\mathrm{g}}$. Other criteria for determining the maximum field-effect mobility give similar results. The experimental errors in $\mu_{\mathrm{e}}$ and $\mu_{\mathrm{h}}$ are determined by the fitting procedure described above; the errors in $V_{\mathrm{g}, \min }, \sigma_{\text {res }}, \Delta V_{\mathrm{g}}$ (plateau width) and $\mu_{\mathrm{e}} / \mu_{\mathrm{h}}$ are then calculated using equation (1) and standard error propagation. The errors (standard deviation) in $\mu_{\mathrm{e}}, \mu_{\mathrm{h}}$ and $V_{\mathrm{g}, \min }$ were typically less than $4 \% . \sigma_{\min }$ is measured directly, and has less than $1 \%$ error. Error bars ( \pm one standard deviation) are shown in Fig. 3, inset for the errors in $\mu_{\mathrm{e}} / \mu_{\mathrm{h}}$, and in Fig. 5 for the errors in $\sigma_{\text {res }}$ and $\Delta V_{\mathrm{g}}$. The weighted mean of $\mu_{\mathrm{e}} / \mu_{\mathrm{h}}$ at non-zero dosing time is 0.83 and the weighted standard deviation of the mean is 0.01 .

\section{Received 20 August 2007; accepted 3 March 2008; published 13 April 2008.}

\section{References}

1. Novoselov, K. S. et al. Electric field effect in atomically thin carbon films. Science 306, 666-669 (2004)

2. Novoselov, K. S. et al. Two-dimensional gas of massless Dirac fermions in graphene. Nature 438, 197-200 (2005)

3. Tan, Y.-W. et al. Measurement of scattering rate and minimum conductivity in graphene. Phys. Rev Lett. 99, 246803 (2007)

4. Chen, J.-H. et al. Printed graphene circuits. Adv. Mater. 19, 3623-3627 (2007).

5. Zhang, Y., Tan, Y.-W., Stormer, H. L. \& Kim, P. Experimental observation of the quantum Hall effect and Berry's phase in graphene. Nature 438, 201-204 (2005).

6. Cho, S. \& Fuhrer, M. S. Charge transport and inhomogeneity near the minimum conductivity point in graphene. Phys. Rev. B 77, 084102(R) (2008).

. Ando, T. Screening effect and impurity scattering in monolayer graphene. J. Phys. Soc. Jpn 75, 074716 (2006).

8. Nomura, K. \& MacDonald, A. H. Quantum transport of massless Dirac fermions. Phys. Rev. Lett. 98, 076602 (2007).

9. Ziegler, K. Robust transport properties in graphene. Phys. Rev. Lett. 97, 266802 (2006).

10. Peres, N. M. R., Guinea, F. \& Castro Neto, A. H. Electronic properties of disordered two-dimensional carbon. Phys. Rev. B 73, 125411 (2006)

11. Kim, E.-A. \& Castro Neto, A. H. Graphene as an electronic membrane. Preprint at $<$ http://xxx.lanl.gov/abs/cond-mat/0702562> (2007).

12. Katsnelson, M. I. \& Geim, A. K. Electron scattering on microscopic corrugations in graphene. Phil. Trans. R. Soc. A 366, 195-204 (2008).

13. Cheianov, V. V. \& Fal'ko, V. I. Friedel oscillations, impurity scattering, and temperature dependence of resistivity in graphene. Phys. Rev. Lett. 97, 226801 (2006).

14. Hwang, E. H., Adam, S. \& Das Sarma, S. Carrier transport in two-dimensional graphene layers. Phys. Rev. Lett. 98, 186806 (2007)

15. Adam, S., Hwang, E. H., Galitski, V. M. \& Das Sarma, S. A self-consistent theory for graphene transport. Proc. Natl Acad. Sci. USA 104, 18392 (2007).

16. Novikov, D. S. Numbers of donors and acceptors from transport measurements in graphene. Appl. Phys. Lett. 91, 102102 (2007).

17. Trushin, M. \& Schliemann, J. The minimum electrical and thermal conductivity of graphene: Quasiclassical approach. Phys. Rev. Lett. 99, 216602 (2007).

18. Yan, X.-Z., Romiah, Y. \& Ting, C. S. Electric transport theory of Dirac fermions in graphene. Preprint at $<$ http://xxx.lanl.gov/abs/0708.1569> (2007)

19. Ishigami, M., Chen, J. H., Cullen, W. G., Fuhrer, M. S. \& Williams, E. D. Atomic structure of graphene on $\mathrm{SiO}_{2}$. Nano Lett. 7, 1643 (2007).

20. Fradkin, E. Critical behavior of disordered degenerate semiconductors. I. Models, symmetries, and formalism. Phys. Rev. B 33, 3257-3262 (1986).

21. Ludwig, A. W. W., Fisher, M. P. A., Shankar, R. \& Grinstein, G. Integer quantum Hall transition: An alternative approach and exact results. Phys. Rev. B 50, 7526 (1994).

22. Ferrari, A. C. et al. Raman spectrum of graphene and graphene layers. Phys. Rev. Lett. 97 , 187401 (2006).

23. Sjovall, P. Intercalation of potassium in graphite studied by thermal desorption spectroscopy. Surf. Sci. 345, L39-L43 (1996).

24. Schedin, F. et al. Detection of individual gas molecules adsorbed on graphene. Nature Mater. 6, $652-655$ (2007).

25. Hwang, E. H., Adam, S. \& Das Sarma, S. Transport in chemically doped graphene in the presence of adsorbed molecules. Phys. Rev. B 76, 195421 (2007).

26. Caragiu, M. \& Finberg, S. Alkali metal adsorption on graphite: A review. J. Phys. Condens. Matter 17, R995-R1024 (2005). 
27. Dresselhaus, M. S. \& Dresselhaus, G. Intercalation compound of graphite. Adv. Phys. 30, 139 (1981). 28. Ziambaras, E., Kleis, J., Schroder, E. \& Hyldgaard, P. Potassium intercalation in graphite: A van der Waals density-functional study. Phys. Rev. B 76, 155425 (2007).

29. Rutter, G. M. et al. Scattering and interference in epitaxial graphene. Science 317, 219 (2007).

30. Novoselov, K. S. et al. Two-dimensional atomic crystals. Proc. Natl Acad. Sci. 102, 10451-10453 (2005)

Supplementary Information accompanies this paper on www.nature.com/naturephysics.

Acknowledgements

This work has been supported by the Laboratory for Physical Sciences (E.D.W.), the US ONR grant N000140610882 (C.J., M.S.F.), NSF grant CCF-06-34321 (M.S.F.) and NSF-UMD-MRSEC grant
DMR 05-20471 (J.H.C.). M.I. was supported by the Intelligence Community Postdoctoral Fellowship program. We thank S. Beatty and G. Rubloff for use of the micro-Raman spectrometer.

Author contributions

M.I., E.D.W. and M.S.F. conceived the experiments, M.I. designed the experimental apparatus,

J.H.C. and C.J. fabricated devices and performed the bulk of the experiments and data analysis, S.A. aided in the theory and J.H.C., M.I., E.D.W. and M.S.F. cowrote the paper. All authors discussed the results and commented on the manuscript.

\section{Author information}

Reprints and permission information is available online at http://npg.nature.com/reprintsandpermissions. Correspondence and requests for materials should be addressed to M.I. 